\title{
A Comparative Numerical Study on Freeway Weaving Area using a Multi-class First-order Macroscopic Model
}

\author{
Saeed Mohammadian ${ }^{1}$, Abolfazl Mohammadzadeh Moghaddam $^{2}$, Ali Sahaf ${ }^{3}$ \\ ${ }^{1}$ M.S. Student in the Department of Highway and Transportation, Ferdowsi University of Mashhad, Iran \\ ${ }^{2}$ Assistant Professor in the Department of Highway and Transportation, Ferdowsi University of Mashhad, Iran \\ ${ }^{3}$ Assistant Professor in the Department of Highway and Transportation, Ferdowsi University of Mashhad, Iran \\ Mohammadians@stu-um.ac.ir; AB-moghaddam@um.ac.ir; asahaf@um.ac.ir
}

\begin{abstract}
This study investigates the impeding effect of weaving area on freeway traffic flow based on a multi-class first-order macroscopic model proposed by Zhu et al (2002). The model assumes lane-changing movement between neighboring lanes is a function of difference in their density. Free traffic condition in road segment with slight difference in density between lanes was presumed in the freeway and several ramp flows were added to the traffic and the density profile of each lane were compared. Results revealed that with the increase in ramp flows, traffic become instable in highway and interchange movements between lanes also rises. The rest of the papers is organized as follows. In section 1, a general description of problem is proposed and in section 2 the formulation used in this paper is introduced. In section 3 the numerical method used in the study is covered and in the final section results and conclusion of the paper is discussed.
\end{abstract}

Keywords: Freeway-Weaving - Traffic flow-Lane-changing

\section{Introduction}

In Highways, where there is an on-ramp or off-ramp, merging and diverging movements between the vehicles affect the traffic state. Where there is an on-ramp following by off-ramp trajectories of vehicles may intercept which is referred to weaving zone. On-ramps add extra traffic demand on the highways and off-ramps due to mandatory lane-changing of vehicles pose backward shock to the traffic stream both of which may make traffic instable. Especially when the level of service is high, these interferences made by weaving area is of great importance. In the rest of the paper, described situation is studied through a numerical simulation and the effect of weaving area on the traffic situation is investigated.

Consider a highway segment as shown in Figure 1, where there are four bands, in which, there is an on-ramp at the position $x=250$, while there is an off-ramp at the position $x=750 \mathrm{~m}$. Typically, vehicles could be divided into 4 groups: A) those, which are in the highway and will drive in the straight part, B) a group of vehicles that change rout from highway to the off-ramp, C) those, which are in the on-ramp and will drive to the highway and finally D) a part of vehicles that will drive from on-ramp to the off-ramp. There is no conflict between the trajectory of vehicles of group A (those driving in the highway) and group D (vehicles travelling from on-ramp to the off-ramp), however, vehicles of group C (departing from highway to the on-ramp) and group $\mathrm{D}$ (those travelling from on-ramp to the highway) may make movements that crosses the other groups' path and their trajectories may intercept.

It is assumed that route choice has been made before this area of the highway. Thus, drivers in the lane 3 and 4 will not drive to the off-ramp. Also, it is a plausible assumption that vehicles travelling from the on-ramp to the off-ramp make no lane-changes during passing acceleration and deceleration lane. However, vehicles of group B and V will make mandatory lane-changes and because of their decreased velocity, the density of lane 1 and possibly lane 2 will raise. This increase in density in lane 1 and 2 will result in inevitable lane-changing of vehicles driving in the highway but not coming from onramp and will not travel to the off-ramp. Velocity, delay time and lateral movements of vehicles differ depending on the initial density of the highway and density and discharge rate of on-ramp. In this paper, road sections will be analyzed under three different scenarios and results will be compared. At first, formulation for freeway containing weaving area is covered. In the succeeding section, adapted numerical method is fully elaborated on and finally consequent results and discussions are provided. 


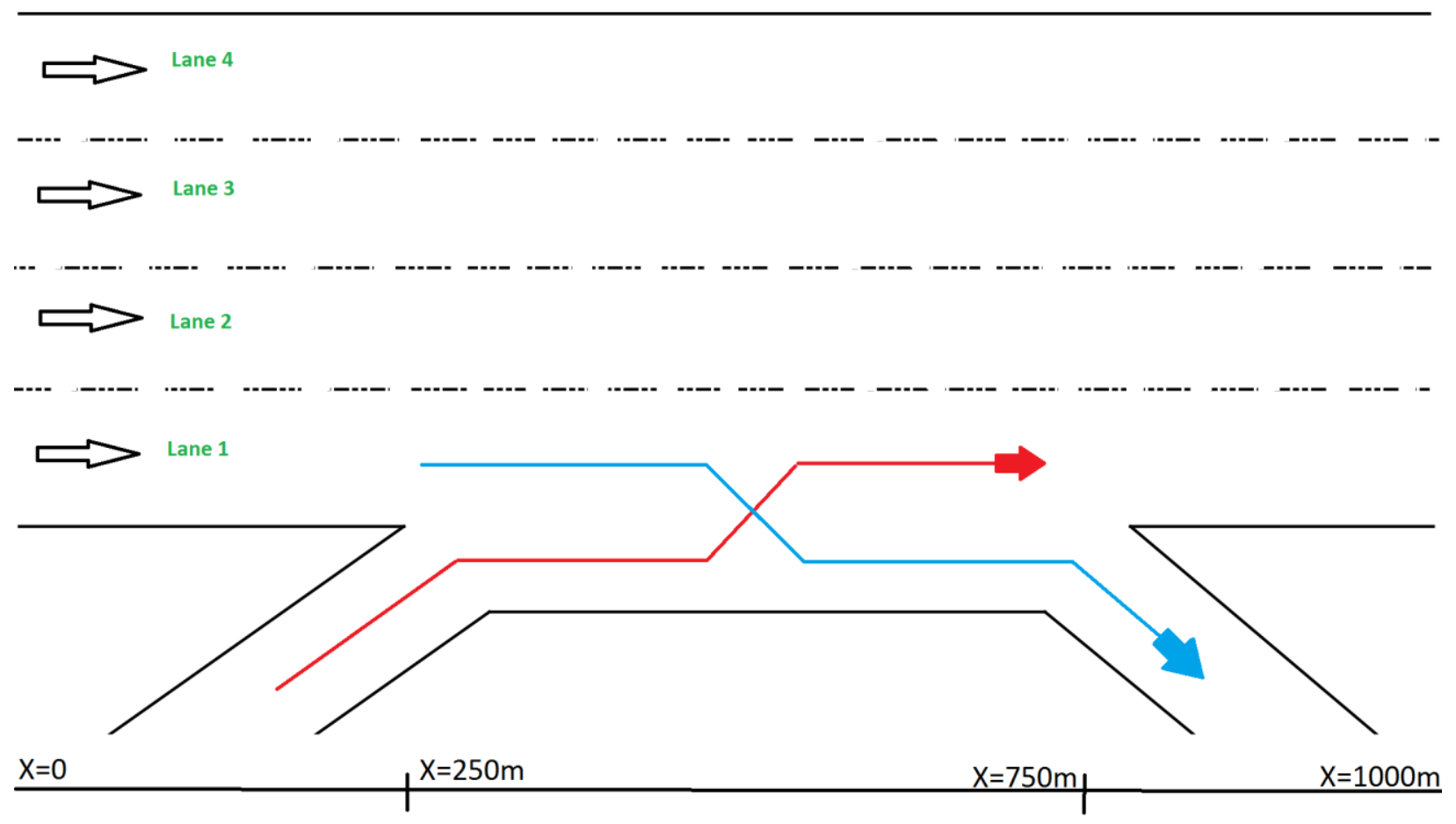

Fig. 1: A Section of highway containing weaving area.

\section{Formulation}

The 3 following premises form the basis of a prospective traffic flow interactions model at a lane closure segment in a highway:

1) The difference in traffic density between adjoining lanes leads to lane-changing movement (Michalopoulos et al., 1984).

2) Flow capacity and jam density are identical for each lane.

3) The effect of on-ramp and off-ramp on the freeway can be regarded as a source and sink on lane 1.

In order to ease the presentation of the proposed model, some notions will be defined. $\Delta x$ is the unit of length and $t_{0}=\frac{\rho_{m} \Delta x}{q_{0}}$ represents time. Also, the total approaching flow is normalized with the lane capacity, $q_{0}$ and so is density with jam density $\left(\rho_{m}\right)$. Thus, according to the conservation relation, one can have the following initial four-lane model for the freeway segment in the absence of ramps.

$$
\left\{\begin{array}{c}
\frac{\partial \rho_{1}}{\partial t}+\frac{\partial q_{1}}{\partial x}=\alpha f\left(\Delta \rho_{1,2}, \beta\right)\left(\rho_{2}-\rho_{1}\right)+v_{\text {ramp }} \\
\frac{\partial \rho_{2}}{\partial t}+\frac{\partial q_{2}}{\partial x}=-\alpha f\left(\Delta \rho_{1,2}, \beta\right)\left(\rho_{2}-\rho_{1}\right)+\alpha f\left(\Delta \rho_{2,3}, \beta\right)\left(\rho_{3}-\rho_{2}\right) \\
\frac{\partial \rho_{3}}{\partial t}+\frac{\partial q_{3}}{\partial x}=-\alpha f\left(\Delta \rho_{2,3}, \beta\right)\left(\rho_{3}-\rho_{2}\right)+\alpha f\left(\Delta \rho_{3,4}, \beta\right)\left(\rho_{4}-\rho_{3}\right) \\
\frac{\partial \rho_{4}}{\partial t}+\frac{\partial q_{4}}{\partial x}=-\alpha f\left(\Delta \rho_{3,4}, \beta\right)\left(\rho_{4}-\rho_{3}\right)
\end{array}\right.
$$

Where:

$\alpha$ : a parameter for defining the lane-changing rate, normalized by $\frac{1}{t_{0}}$ and in this paper the value 0.05 has been adopted for the study.

$\beta$ : maximum normalized difference in density between adjoining lanes, used to define $f(\Delta \rho, \beta)$ 
$f(\Delta \rho, \beta)$ : Lane-changing modifier with $\Delta \rho_{1,2}=\left(\rho_{1}-\rho_{2}\right)$, and $\Delta \rho_{2,3}=\left(\rho_{2}-\rho_{3}\right)$, and $\Delta \rho_{3,4}=\left(\rho_{4}-\rho_{3}\right)$

$$
v_{\text {ramp }}=\left\{\begin{array}{cc}
\frac{Q_{\text {ramp }}}{L_{\text {on-ramp }}}, & \text { in onramp influence area } \\
-\frac{Q_{\text {ramp }}}{L_{\text {on-ramp }}}, \quad \text { in offramp influence area } \\
0 \quad \text { in the other parts of highway }
\end{array}\right.
$$

Where

$Q_{\text {ramp }}$ is the flow rate in on-ramp and off-ramp and $v_{\text {ramp }}$ is density of flow rate over length of ramp acceleration or deceleration lane.

Based on the hyperbolic model for non-Newtonian fluid dynamics in porous media (Mychidinov et al., 1989), the modifier can be expressed as follows:

$$
f(\Delta \rho, \beta)=\frac{|\Delta \rho|}{\beta+\sqrt{1+|\Delta \rho|^{2}}}
$$

As one can observe in Figure 2, differences in density play a recognizable role in the result of $f(\Delta \rho, \beta)$ function. In this study $\beta=0.1$ has been adopted because it is a mid-range value.

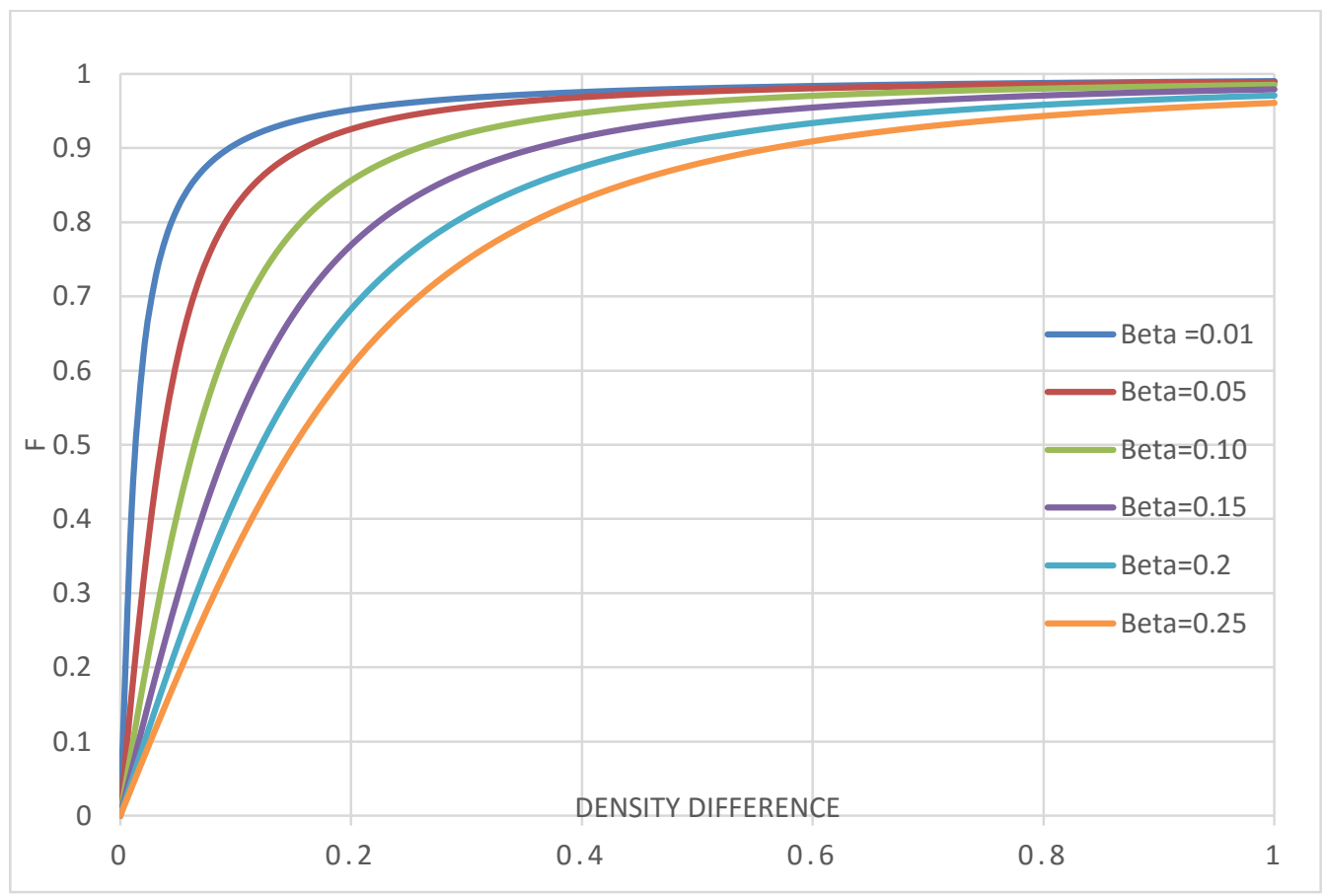

Fig. 2: The Variable Dependence of the Function $f(\Delta \rho, \beta)$.

Zhu et al (2002), proposed a single parameter equation for flow rate as follows:

$$
q_{i}=q_{0} \cdot\left(1+A_{i}\left(\rho_{i}-b_{i}\right)^{2}+B_{i}\left(\rho_{i}-b_{i}\right)^{3}\right), i=1,2,3,4
$$

Where:

$b_{i}=\rho_{0, i} / \rho_{m}:$ Normalized optimal density for the $i$-th lane, and $q_{0}:$ Maximum capacity for each lane 


$$
A_{i}=-\frac{\left(1-b_{i}\right)^{3}+b_{i}{ }^{3}}{b_{i}{ }^{2}\left(1-b_{i}\right)^{2}} \text { and } B_{i}=\frac{\left(1-b_{i}\right)^{2}-b_{i}{ }^{2}}{b_{i}{ }^{2}\left(1-b_{i}\right)^{2}}, i=1,2,3,4
$$

Figure 3 illustrates the relationship between normalized rate of flow and normalized density for several values of $b$ for a single lane.

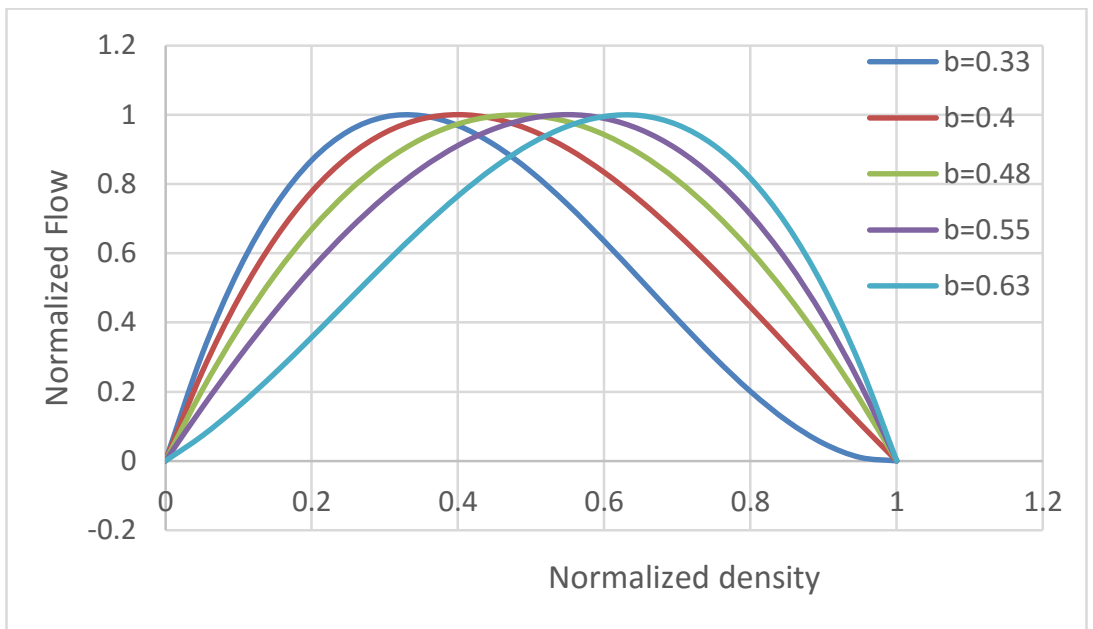

Fig. 3: Relationship between Flow Rate and Density for Several Values of $b$.

In this study $b=0.33$ is chosen for traffic state in freeway. This is because recent researches have shown that in most cases fundamental diagram for flow and density is more similar to that of $b=0.33$ (Treiber, 2012) and adopting this value for $b$, equation 3 can simplified to the following equation.

$$
q_{i}=q_{0} \cdot\left(1-6.88\left(\frac{\rho_{i}}{\rho_{m}}-0.33\right)^{2}+6.95\left(\frac{\rho_{i}}{\rho_{m}}-0.33\right)^{3}\right)
$$

\section{Numerical Solution and Initial Conditions}

The above weaving area traffic model is solved with upwind method and mesh-grid in time and space as follows:

$$
\begin{cases}\rho_{i}^{n+1}=\rho_{i}^{n}-\frac{\Delta t}{\Delta x}\left(q_{i}^{n}-q_{i-1}^{n}\right)+\Delta t . s & \text { if } \frac{d q}{d \rho}>0 \\ \rho_{i}^{n+1}=\rho_{i}^{n}-\frac{\Delta t}{\Delta x}\left(q_{i+1}^{n}-q_{i}^{n}\right)+\Delta t . s & \text { if } \frac{d q}{d \rho}<0\end{cases}
$$

Where:

$\Delta x$ : Length of the spatial step which is set to be $10 \mathrm{~m}$.

$\Delta t$ : Temporal step which is set to be $0.1 \mathrm{~s}$ and 500 temporal iteration has been accomplished.

$\rho_{i}^{n}$ : Density of i-th segment in the nth time step

$q_{i}^{n}$ : Flux (flow rate) in the i-th segment and nth time step

$\mathrm{s}$ : the source term for lane-changing movement between lanes. For lane 1 and within weaving area, source term also contains $v_{\text {ramp }}$ which is flow rate from on-ramps and off-ramp

$\frac{d q}{d \rho}$ : Characteristic speed of flow rate and represents traffic wave 
- Numerical stability analysis

The first CFL condition which is defined as below is checked through the numerical solution of the problem. The first CFL condition is about the size of the mesh-grids in the discretization and is stated as below

$$
C=\frac{u_{\max } d t}{d x} \leq C_{\max }
$$

Where

$C$ : the courant number

$u_{\max }$ : Maximum Velocity

$d t$ : Temporal element size in each step

$d x$ : Spatial element size in each step

$C_{\text {max }}$ : Maximum value of courant number

Maximum courant number is usually and in this paper set to be 1 and using presented values for other parameters it is clear that CFL condition is satisfied.

\section{- Initial conditions and assumptions}

$\checkmark$ The highway profile is $1000 \mathrm{~m}$ and the on-ramp position is at $\mathrm{x}=250 \mathrm{~m}$ whereas the off-ramp position is at $\mathrm{x}=750 \mathrm{~m}$

$\checkmark$ Maximum rate of flow is set to be $2200 \mathrm{veh} / \mathrm{hr} /$ lane and maximum density is set on $150 \mathrm{veh} / 1000 \mathrm{~m}$

$\checkmark \quad$ stochastic lateral movement of drivers is neglected and lane-changing is limited to traffic pressure caused by difference in density or route changing within weaving area

$\checkmark$ Initial density of each lane is set to be within range of [0.14 $\rho_{\text {jam }} 0.28 \rho_{\text {jam }}$ ] so that free traffic condition happens in freeway (with the densities more than $0.33 \rho_{\text {jam }}$ based on the model traffic condition will become unstable), sensitivity analysis has been accomplished with change in flow rate of ramps.

\section{Results and Discussions}

In this study, the effect of weaving area on traffic flow was investigated in terms of change in density profile of each lane. As the flow in on-ramp and off-ramp increased, more lane underwent the perturbation caused by mandatory lanechanging in weaving area. Under free traffic and steady-state flow condition in freeway segment and different ramp flows, several scenario may happen.

As illustrated in Figure 4, when there is no ramp flow, traffic maintains its steady-state situation, however, difference in initial density of lanes lead to gradual changes in density profile of each lane up to equilibrium point.

Figure 5 through Figure 8 show density profile of each lane of the freeway for different values of flow in on-ramp and off-ramp. It is obvious from figures that as flow in both ramps increases, traffic condition in lane 2 and 3 will not be steadystate anymore. Lane 4, however, does not experience a non-uniform density profile unless the flow in ramp is near the capacity.

This study explored the impeding effect of weaving area on a freeway with free traffic condition for several ramp flow values. Lane-changing and other aspect of weaving could be conceived in terms of the change in density of lane. For further research simulation of weaving area contemplating the effect of heavy vehicles on traffic is proposed. It is also proposed to simulate traffic flow condition with second-order models.

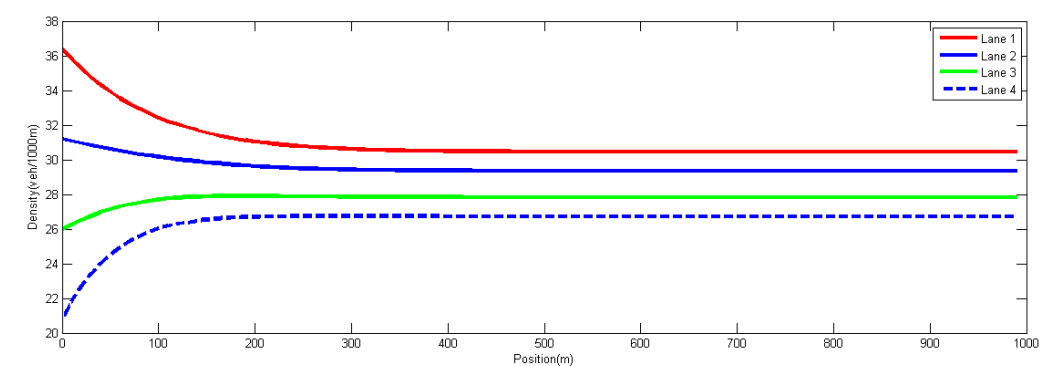

Fig. 4: Density Profile of Freeway Segment-no In-flow and Out-flow from Ramps. 


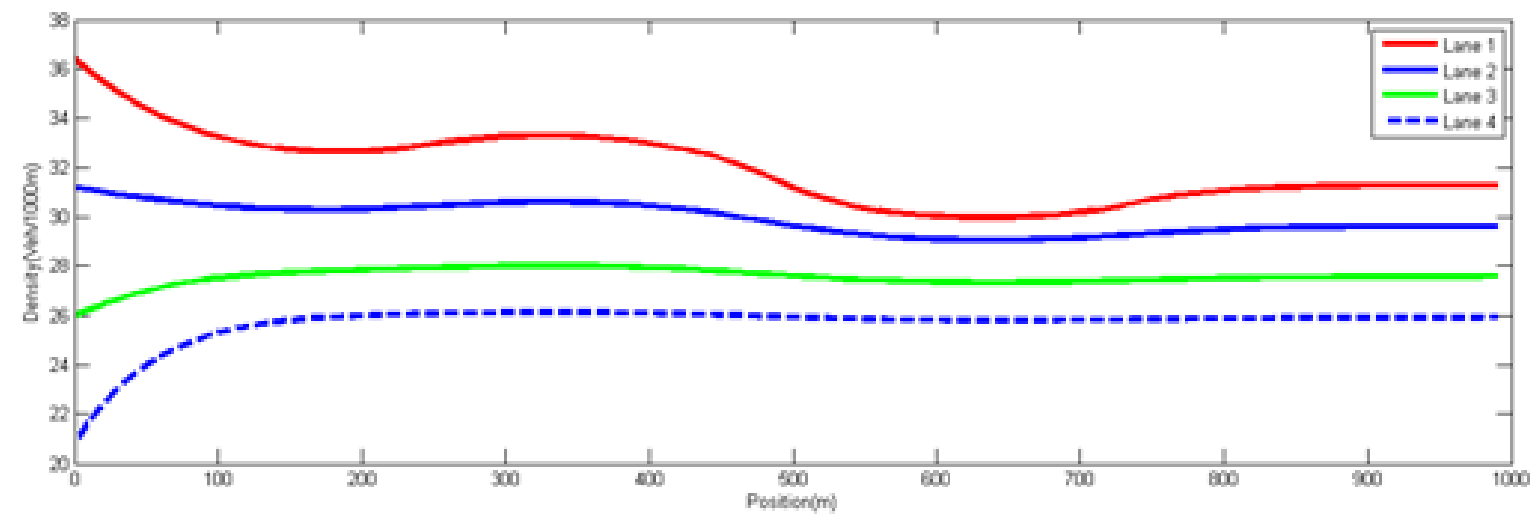

Fig. 5: Density Profile of Freeway Segment-slight flow in Ramps.

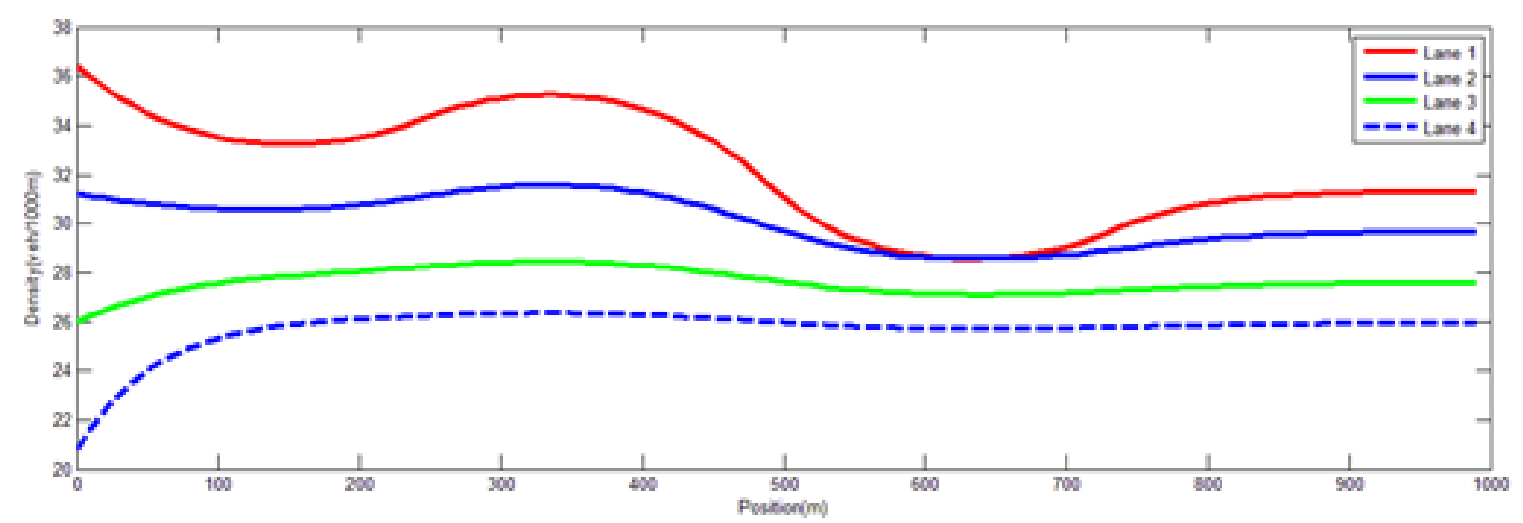

Fig. 6: Density Profile of Freeway Segment- $\mathrm{q}_{\text {on-ramp }}=0.25 \mathrm{q} 0$ and $\mathrm{q}_{\text {off-ramp }}=0.125 \mathrm{q} 0$.

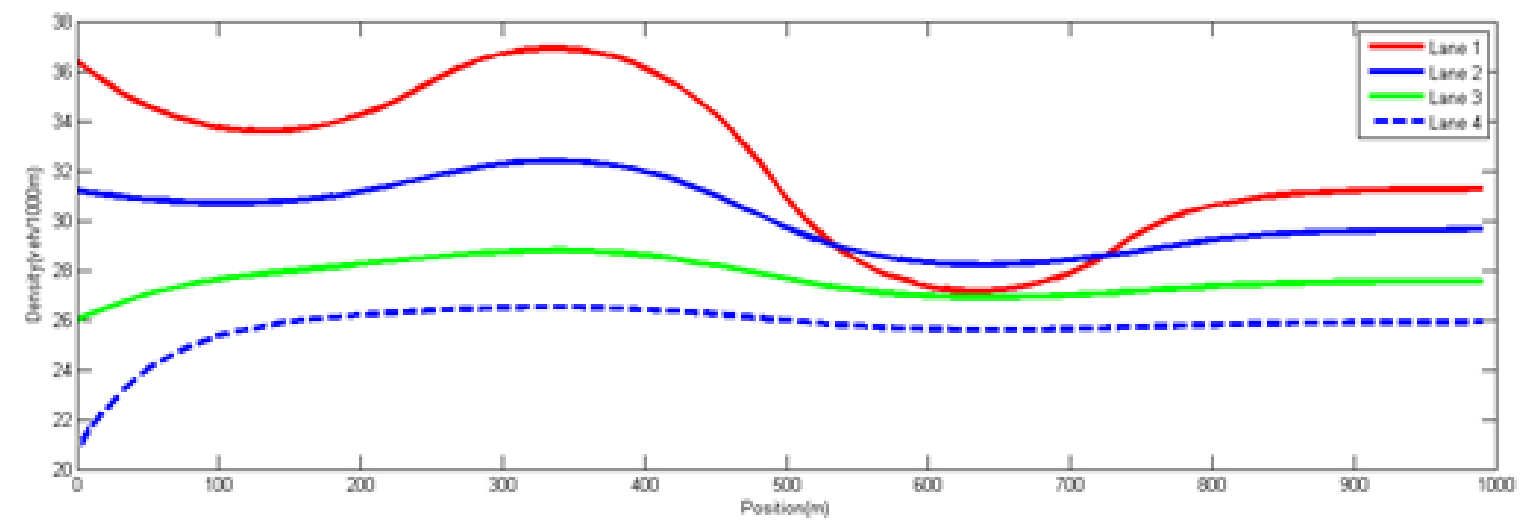

Fig. 7: Density Profile of Freeway Segment- - $\mathrm{q}_{\mathrm{on}-\mathrm{ramp}}=0.36 \mathrm{q} 0$ and $\mathrm{q}_{\text {off-ramp }}=0.18 \mathrm{q} 0$. 


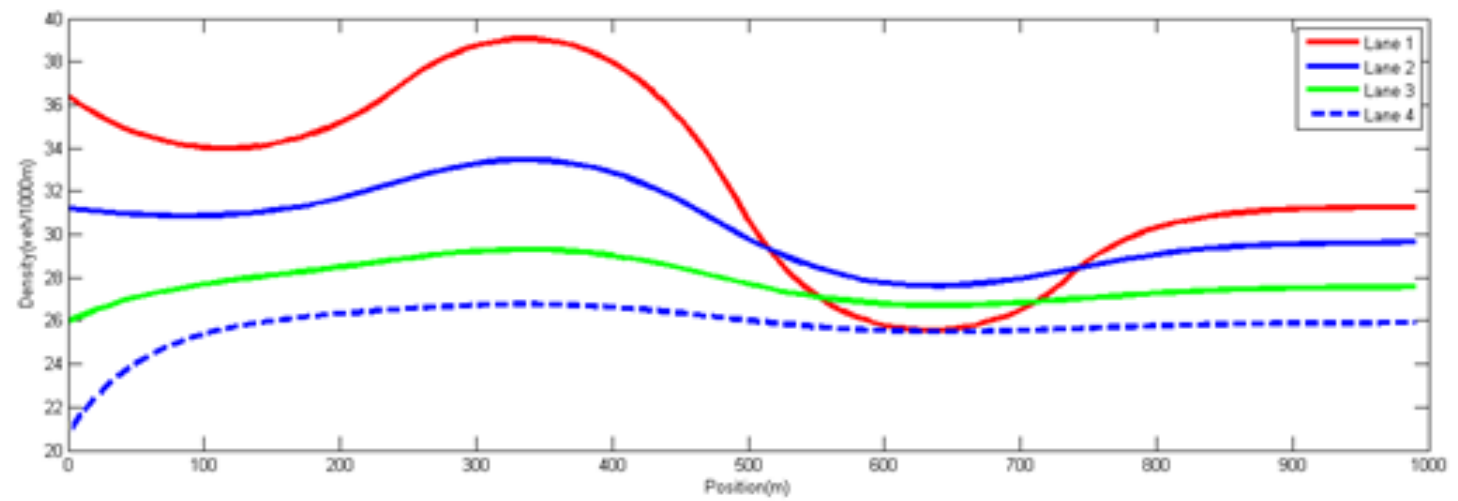

Fig. 8: Density Profile of Freeway Segment- $\mathrm{q}_{\text {on-ramp }}=0.4 \mathrm{q} 0$ and $\mathrm{q}_{\text {off-ramp }}=0.2 \mathrm{q} 0$.

\section{References}

[1] G. L. Chang and Z. J. Zhu, "A Macroscopic Traffic Model for Highway Work Zones, Formulations and Numerical Results," Journal of Advanced Transportation, vol. 40, no. 3, pp. 265-287, 2005.

[2] M. Treiber and A. Kesting, "Traffic flow dynamics, Data, Models, and simulation," in Chapter 8, 2012, pp. 99-105.

[3] J. H. Bick and G. F. Newell, "A continuum model for traffic flow on an undivided highway," Q. Appl. Math., vol. 18, no. 2, pp. 191-204, 1960.

[4] M. J. Lighthill and G. B. Whitham, "On kinematic waves. II: A theory of traffic flow on long crowded roads," in Proc. R. Soc., London, Ser. A, 1955, vol. 229, pp. 317-345.

[5] H. S. Shui, Finite difference in one dimensional fluid mechanics. Beijing: National Defense, 1998. 